\title{
Insights on Sample Topography in EDX Spectroscopy with Annular SDD Detectors
}

\author{
A.Schöning ${ }^{1}$, R.Lackner ${ }^{1}$, A.Bechteler ${ }^{1}$, A.Liebel ${ }^{1}$, J.M.Davis ${ }^{1}$, A.Niculae ${ }^{1}$ and H.Soltau ${ }^{1}$ \\ 1. PNDetector GmbH, München, Germany
}

Energy Dispersive X-ray spectroscopy (EDS) is an indispensable tool in electron microscopy. While EDS analysis of flat samples is easily done, X-ray mapping of topographic samples remains challenging. In many cases, the combination of X-ray shadowing and fluorescence induced by secondary radiation produces misleading X-ray images. In order to evaluate such results, more information is needed to completely understand the actual element distribution of a sample. Fast X-ray imaging over large areas with complex topography requires large area, multichannel detectors in close proximity to the sample in order to get spatial information while covering a large solid angle.

The "Rococo2" Silicon Drift Detector (SDD) has an advanced geometry allowing positioning close to the sample while having a large detector area. It consists of four $15 \mathrm{~mm}^{2}$ SDDs arranged evenly around a center hole, all combined on one monolithic chip. The detector can be positioned right below the pole piece (Figure 1) while the electron beam is guided through the center hole. This arrangement enables a very close proximity to the sample resulting in a solid angle of up to $1.2 \mathrm{sr}$ [1]. The next generation "Rococo 3" SDD makes further improvements in geometry and performance. The chip has four $20 \mathrm{~mm}^{2}$ SDDs also arranged around a center hole. Due to a further optimized geometry, the solid angle reached by the system is now up to $1.8 \mathrm{sr}$. In comparison to a common $10 \mathrm{~mm}^{2}$ SDD with a typical solid angle of about $0.01 \mathrm{sr}$, the Rococo detectors can deliver a hundred times higher signal intensity and therefore reduces the measurement time significantly. The four individual SDD cells deliver a cutting edge energy resolution down to $124 \mathrm{eV}$ FWHM at $\mathrm{Mn}-\mathrm{K} \alpha$ at an operating temperature of $-20{ }^{\circ} \mathrm{C}$. Backscattered electrons are stopped within thin filter foils. By choosing the right filter materials light element performance with high energy resolution can be realized.

The four cells of the Rococo combined provide a very high count rate, but analyzing each signal separately delivers additional spatial information. Having the detector right below the pole piece results in a high pick up angle and therefore minimizes shadowing. This is shown in Figure 2 where high resolution EDS maps of a Tin on Carbon sample were recorded using a conventional $10 \mathrm{~mm}^{2} \mathrm{SDD}$ and the Rococo3 detector at the same conditions and acquisition time. Beyond the higher overall count rate compared to the $10 \mathrm{~mm}^{2} \mathrm{SDD}$, comparing the individual cells of the Rococo one can observe that shadowed areas on one cell are clearly visible on at least one of the others. This not only has the benefit of analyzing the whole sample without rotating it, but also gives an advantage when it comes to the interpretation of topographic samples.

In summary, the Rococo Detector System can cover a large solid angle while providing excellent energy resolution and minimizing shadowing effects. Looking at each of the four cells individually offers valuable information about the topography of the samples. The compact system with integrated preamplifier makes it easy to adapt it to various electron microscopes.

We will present further results with the Rococo Detector System demonstrating the advantages in analyzing topographic samples. 
References:

[1] A Niculae et al, Microscopy \& Microanalysis 18 (S2) (2012), p. 1202.
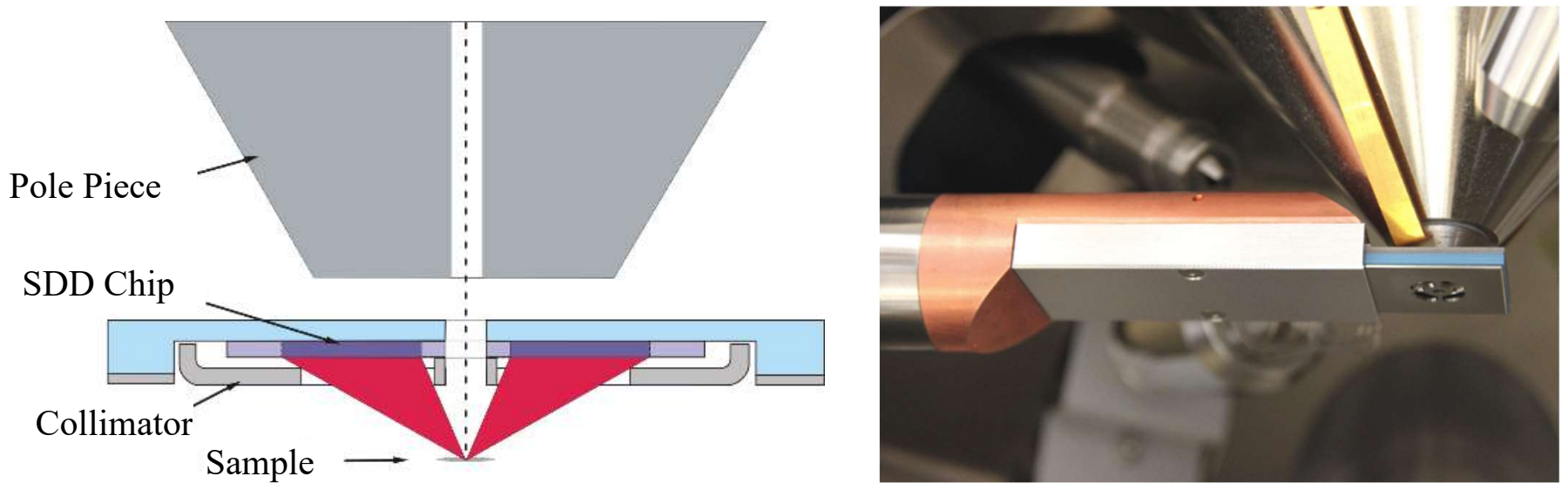

Figure 1. Schematic drawing of the Rococo EDS detector positioned below the pole piece (left). A picture of the detector system inside a SEM (right).
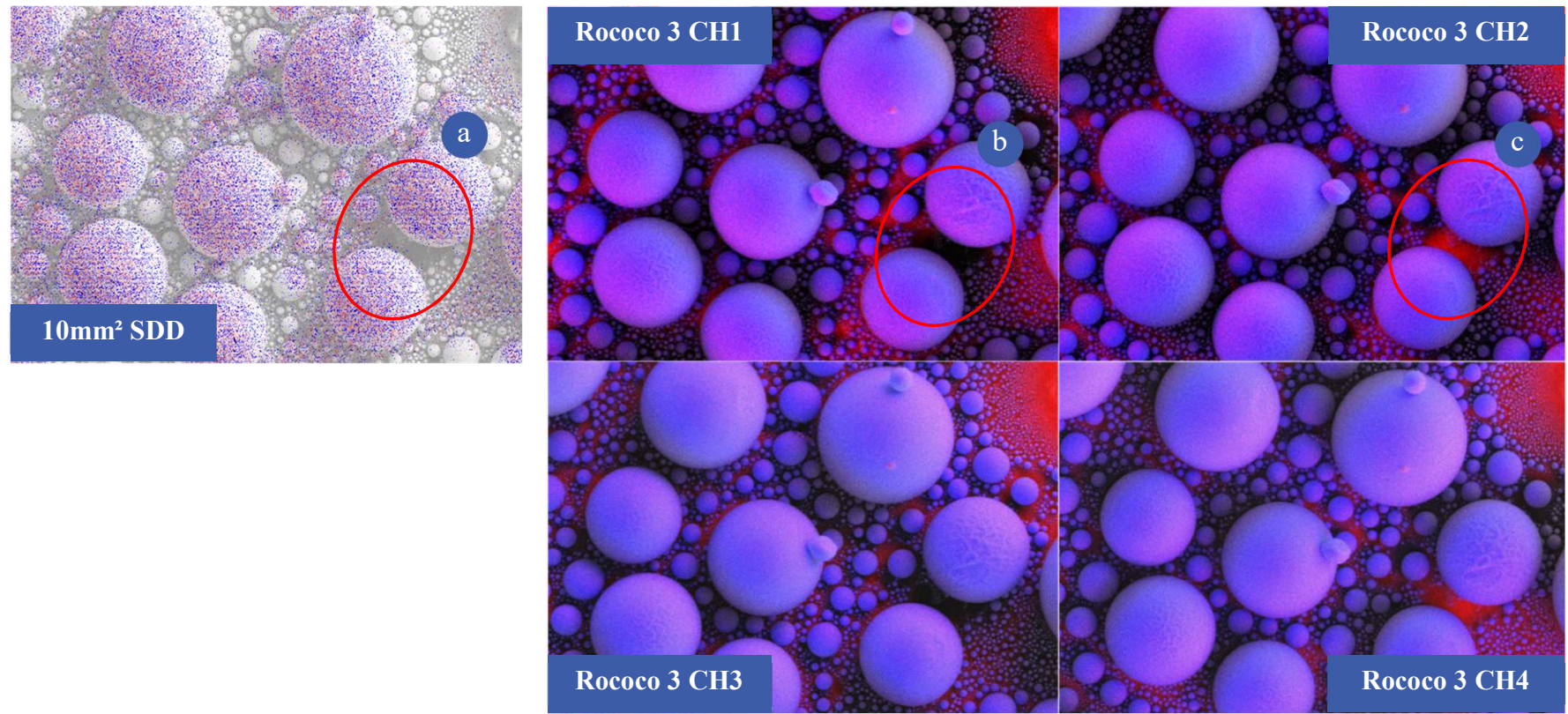

Figure 2. High resolution SEM-EDS maps (1024 x 768) of a Tin on Carbon sample with a conventional $10 \mathrm{~mm}^{2}$ SDD (approx. $0.01 \mathrm{sr}$, left) and a Rococo 3 detector (approx. $1.8 \mathrm{sr}$, right). Measurements were performed with $77 \mathrm{pA}$ beam current, $10 \mathrm{kV}$ beam energy and $60 \mathrm{~min}$ acquisition time. While some of the shadowed areas on the $10 \mathrm{~mm}^{2}$ SDD map (a) also appear on some channels of the Rococo 3 maps (b), they are clearly visible on at least one of the other channels (c). 